\title{
Toll-like receptor 3 (TLR3) \\ and the development of type 1 diabetes mellitus
}

Taís Silveira Assmann ${ }^{1,2}$, Letícia de Almeida Brondani ${ }^{1}, 2$, Ana Paula Bouças ${ }^{1,2}$, Luís Henrique Canani ${ }^{2}$, Daisy Crispim ${ }^{1,2}$

${ }^{1}$ Laboratory of Biology of Human Pancreatic Islet, Endocrinology Division, Hospital de Clínicas de Porto Alegre, Universidade Federal do Rio Grande do Sul (UFRGS), Porto Alegre, RS, Brazil 2 Post-graduate Program in Medical Sciences: Endocrinology, UFRGS, Porto Alegre, RS, Brazil

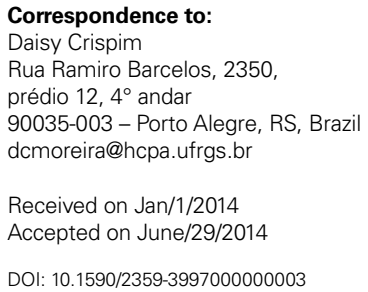

\begin{abstract}
Type 1 diabetes mellitus (T1DM) is a chronic, progressive autoimmune disease characterized by metabolic decompensation often leading to dehydration and ketoacidosis. Viral agents seem to play an important role in triggering the autoimmune destruction that leads to the development of T1DM. Among several viral strains investigated so far, the enterovirus family has been consistently associated with the onset ofT1DM in humans. One of the mediators of viral damage is the doublestranded RNA (dsRNA) generated during replication and transcription of viral RNA and DNA. The Toll-like receptor 3 ( $T L R 3$ ) gene codes for an endoplasmic receptor of the pattern-recognition receptors (PRRs) family that recognizes dsRNA, plays an important role in the innate immune response triggered by viral infection. Binding of dsRNA to the TLR3 triggers the release of proinflammatory cytokines, such as interferons, which exhibit potent antiviral action; thus, protecting uninfected cells and inducing apoptosis of infected ones. Therefore, the TLR3 gene is a good candidate for the development of T1DM. Within this context, the objective of the present review was to address the role of the TLR3 gene in the development ofT1DM. Arch Endocrinol Metab. 2015;59(1):4-12
\end{abstract}

Keywords

Autoimmunity; type 1 diabetes mellitus; viral infection; Toll-like receptor-3 (TLR3)

\section{INTRODUCTION}

$\mathrm{T}$ ype 1 diabetes mellitus (TIDM), which accounts for $5-10 \%$ of all cases of diabetes, is characterized by severe autoimmune destruction of insulin-producing beta-cells in the pancreas by $\mathrm{T}$ lymphocytes and macrophages infiltrating the islets of Langerhans. Consequently, subjects with TIDM are usually dependent on insulin injections for life (1).

Inflammation of the islets (insulitis) probably develops within a context of a "dialog" between immune cells and pancreatic beta-cells. This dialog is mediated by cytokines and chemokines, which are released by immune cells and beta-cells, as well as by other immunogenic signals delivered by dying beta-cells. This may lead to induction and amplification of the inflammatory process, but in some cases, may lead to the resolution of insulitis (2). Furthermore, the course of beta-cell inflammation and its potential progression to clinical TIDM depend on a complex interaction between a strong genetic component and a diversity of environmental triggers $(3,4)$.
Among the several loci associated with TIDM, the human leukocyte antigen (HLA) class II locus is undoubtedly the leading genetic risk factor for TIDM, accounting for $30-50 \%$ of the genetic risk for this disease (5). Other genes are associated with minor effects on TIDM risk when compared with HLA, such as the insulin gene, the cytotoxic T-lymphocyte associated protein 4 (CTLA4) gene, the protein tyrosine phosphatase, nonreceptor type 22 (PTPN22) gene, and other genes discovered through genome-wide association studies (GWAS) $(5,6)$.

Several epidemiologic studies have found that environmental factors, such as viral pathogens operating early in life, seem to trigger the autoimmune destruction of beta-cells in genetically susceptible subjects (7-9). Host defense against invading microbial microorganisms is elicited by the immune system, which consists of two components: innate immunity and adaptive immunity. Both components of immunity recognize invading microorganisms as non-self, thus, triggering immune responses to eliminate them (10). 
Innate immunity is the first line of defense against viruses, bacteria and fungi. Detection of viruses or other invading microorganisms is carried out by a wide range of cell receptors of the pattern-recognition receptor (PRRs) family, which recognize highly conserved pathogen-associated molecular patterns (PAMPs), such as the double-stranded RNA (dsRNA) generated during the life cycle of most viruses $(11,12)$. Innate immune cells, such as macrophages and dendritic cells, kill invading microorganisms through phagocytosis or production of cytokines, chemokines and type I interferons. These interferons (IFNs) have a potent antiviral effect, protecting uninfected cells and inducing apoptosis of those already infected (13). Moreover, activation of innate immunity is an important step in the development of an antigen-specific adaptive immune system, consisting of B lymphocytes, which produce specific antibodies against the invading pathogen, and T lymphocytes, which secrete cytokines that will induce elimination of infected cells by exerting cytotoxic effects or by signaling B lymphocytes (10,12).

Many types of PRRs have been identified so far, including toll-like receptors (TLRs), nucleotide-binding oligomerization domain (NOD)-like receptors (NLRs) and retinoic acid-inducible gene I (RIG-I)-like helicases (RLHs) $(13,14)$. Of these, the most extensively studied are TLRs, which are type I transmembrane glycoproteins originally identified in Drosopbila $(11,15)$. Human homologs are known to comprise at least 10 members with different response triggers (16). Amongst the human TLRs, TLR3 has been shown to respond to dsRNA, a replication product of some virus, and it is expressed at high levels in human and mouse pancreatic beta-cells and in antigen-presenting dendritic cells $(2,17)$. Therefore, TLR3 is a plausible candidate gene for TIDM susceptibility. Within this context, the objective of the present review was to address the role of the TLR 3 gene in the development of TIDM.

\section{VIRUSES AND TYPE 1 DIABETES MELLITUS}

The hypothesis that viral infections are involved in TIDM is based on epidemiological studies and anecdotal data $(9,18)$. One of the major observations that support a role for viruses in TIDM is that concordance rates for TIDM in monozygotic twins are only $\sim 50 \%$ instead of the expected $100 \%$ if the characteristic would be explained only by genetic factors. Also, there was an increase in the TIDM incidence over the past 50 years in many countries, which cannot be explained by alterations in genetic risk factors. In addition, migration studies show that the incidence of TIDM in offspring of subjects who had moved from a low-incidence to a high-incidence area is increased compared to the original incidence observed in the area of origin. Importantly, viruses and virus-specific antibodies can be detected in newly diagnosed patients with TIDM $(8,18,19)$. Indeed, a number of viruses have been associated with TIDM in humans, such as enteroviruses, rubella virus, mumps virus, rotaviruses, parvoviruses, and cytomegalovirus $(18,20)$. Overall, these data demonstrate a correlation between particular virus infections and human TIDM; however, this correlation neither establishes a direct link between microbial infections and the disease nor provides mechanistic insights into the autoimmune process leading to disease (18).

The best documented correlation between a virus and TIDM has been for enteroviruses, non-enveloped single-stranded RNA viruses belonging to the picornavirus family $(8,21)$. Enterovirus infections are more frequent in siblings developing TIDM compared to nondiabetic siblings, and enterovirus antibodies are elevated in pregnant women whose children later develop TIDM (22). Interestingly, epidemiological studies of the seasonality of development of anti-beta-cell antibodies in a group of subjects at increased risk of TIDM showed an increased incidence of autoantibodies during winter, which correlated with a period of increased enteroviral infection rates $(9,20)$. Recently, Oikarinen and cols. (23) isolated enteroviruses from intestinal biopsies in $75 \%$ of TIDM patients and in $10 \%$ of nondiabetic subjects, indicating that a substantial proportion of TIDM patients have an ongoing enterovirus infection in gut mucosa. Elshebani and cols. (24) found that enteroviruses isolated from newly diagnosed TIDM patients could infect human islets in vitro, reducing their ability to secrete insulin in response to high glucose, and increasing the number of dead cells. In addition, a recent meta-analysis, including 24 papers and 2 abstracts, showed a significant association between enterovirus infection and TIDM-related autoimmunity $(\mathrm{OR}=3.7,95 \% \mathrm{CI}=2.1-6.8)$ or clinical $\mathrm{TlDM}(\mathrm{OR}=$ 9.8, 95\% CI $=5.5-17.4)(25)$.

Among the enteroviruses, the most significant association with TIDM was with Coxsackievirus (CVB), which exhibits a specific tropism for the pancreas $(26,27)$. Serological and PCR analyses have shown that CVB are expressed more frequently in TIDM patients 
than in healthy subjects [reviewed in (18)]. One CVB4 strain was isolated from the pancreas of a child who died from diabetic ketoacidosis, and this virus caused diabetes when inoculated into a susceptible mouse strain (28). Furthermore, Dotta and cols. (29) identified CVB4 in $50 \%$ of samples collected from patients with TIDM, and these enteroviruses were able to infect human islets in vitro, impairing insulin secretion in response to glucose.

Based on the studies presented above, enteroviruses appear to be associated with a fraction of TIDM cases. Nevertheless, if enteroviruses play a major role in the TIDM pathogenesis, how could we explain the increase in TIDM incidence in countries where exposure to these microorganisms has been dropping, such as Finland? Do the data show that TIDM can be caused by viral infections compatible with the hygiene hypothesis [see reference (30) for more details]? Interestingly, data in NOD mice revealed that CVB, and the $\mathrm{B} 4$ strain in particular, infect the exocrine portion of the pancreas and provoke diabetes in animals with established insulitis (31). Interestingly, when administered earlier in life, CVB inoculation is associated with a strong protection against diabetes, implying that the timing of the infection plays a key role in disease development (32). Taking into account the studies in NOD mice, Coppieters and cols. (8) suggested that the lack of exposure to enteroviruses in developed countries results in a reduced frequency of subjects with protective immunity through early childhood infections. When islet inflammation occurs in these subjects, they would be more susceptible to an enteroviral infection that has the potential to initiate autoreactivity and beta-cell damage.

Animal models have provided important data about the possible mechanisms of enterovirus-induced betacell damage. They have shown that several mechanisms may be involved and either a direct infection of betacells, molecular mimicry or bystander activation of autoreactive immune clones induce beta-cell damage [see reference (21) for more details]. Studies on human pancreata or cultured islets have shown that there are considerable variations in the adverse effects of enteroviruses on beta-cells, not only between various viral serotypes, but also between strains of the same serotype (20). Viral infections might be able to "unmask" betacells for recognition by $\mathrm{CD} 8^{+} \mathrm{T}$-cells by promoting IFN production and upregulation of MHC class I molecules on beta-cells. These events may be sufficient to mark pancreatic islets for autoimmune attack (20). However, the precise mechanism by which viruses cause TIDM in humans remains to be determined.

\section{TOLL-LIKE RECEPTORS (TLRS)}

Among the most important families of PRRs are the TLRs which selectively recognize a large number of PAMPs derived from microbes (15). Mammalian TLRs also respond to host-derived molecules that are released from injured tissues and cells, termed damage associated molecular patterns (DAMPs) (33). TLR-ligand binding plays a key role in innate immunity and subsequent acquired immunity against microbial infection or tissue injury (34).

Structurally, TLRs are type I transmembrane-signaling PRRs. Their extracellular domain includes a repetitive structure rich in leucine residues, called leucine-rich repeat (LRR), which is involved in ligand recognition. A transmembrane domain determines their cellular localization, and the intracellular portion contains a similar structure to that of the IL-1 receptor, known as Toll/IL-l (TIR) domain, which is essential for triggering downstream signaling pathways (35-37). So far, ten TLRs have been identified in humans and 13 in mice, recognizing different PAMPs and DAMPs $(12,13,36)$. Table 1 shows the 11 human TLRs with their specific ligands. The ligands for TLR8 and TLRI0 remain unknown (38). The human TLRII gene has a stop codon within an open reading frame and thus appears to be non-functional (39).

TLRs are located either on the cell surface or in intracellular compartments. Cell-surface TLRs, including TLR1, TLR2, TLR4, TLR5, TLR6 and TLR10, are essential to recognizing bacterial cell wall components, bacterial flagellin, viral particles, and other unidentified pathogenic components. TLR3, TLR7, TLR8, and TLR9 are localized in endosomes, and their ligands, mainly bacterial or viral nucleic acids, require internalization to the endosome before signaling is possible (Table 1) $(10,34,40)$.

TLRs are widely expressed in various immune cells, including dendritic cells, macrophages, B cells, specific types of $\mathrm{T}$ cells, and even in non-immune cells, such as epithelial cells, endothelial cells, and fibroblasts $(41,42)$. Expression of TLRs is not static but regulated rapidly in response to pathogens, a variety of cytokines, and environmental stresses (42). Interestingly, expression of TLRs in non-immune cells appears to play an important role in the first line of defense against microbial invasions at these sites (35). 
Table 1. Pathogen-associated molecular pattern (PAMPs) detection by toll-like receptors (TLRs)

\begin{tabular}{|c|c|c|c|c|}
\hline TLRs & Localization & Microorganism & PAMPs recognized & $\begin{array}{l}\text { Other PRRs involved in } \\
\text { recognition }\end{array}$ \\
\hline TLR1 & Plasma membrane & Bacteria & Triacyl lipoprotein & \\
\hline \multirow[t]{3}{*}{ TLR2 } & Plasma membrane & Bacteria & Lipoprotein & \\
\hline & & Fungi & Zymosan, beta-glucan & Dectin-1, NALP-3 \\
\hline & & Parasites & Tgpl-mutin (Trypanosoma) & \\
\hline TLR2/1 TLR2/6 & Plasma membrane & $\begin{array}{l}\text { Lipoproteins, LTA, PGN, } \\
\text { lipoarabinomannan }\end{array}$ & Bacteria, mycobacteria & NOD1, NOD2, NALP3, NALP1 \\
\hline TLR3 & Endolysossome & Viruses & dsRNA & RIG-1, MDA5, NALP3 \\
\hline \multirow[t]{4}{*}{ TRL4 } & Plasma membrane & Bacteria & LPS & \\
\hline & & Viruses & Structural protein & \\
\hline & & Parasites & $\begin{array}{l}\text { Glycoinositolphospholipids } \\
\text { (Trypanosoma sp.) }\end{array}$ & \\
\hline & & Fungi & Mannan & \\
\hline TLR5 & Plasma membrane & Bacteria & Flagelin & IPAF, NAIP5 \\
\hline \multirow[t]{3}{*}{ TLR6 } & Plasma membrane & Bacteria & Diacyl lipoprotein & \\
\hline & & Viruses & Diacyl lipoprotein & \\
\hline & & Fungi & Zymosan, beta-glucan & Dectin-1, NALP-3 \\
\hline \multirow[t]{4}{*}{ TLR7 } & Endolysossome & Viruses & ssRNA & RIG-1, MDA5, NALP3 \\
\hline & & Bacteria & RNA & NALP3 \\
\hline & & Self & & \\
\hline & & Fungi & RNA & \\
\hline TLR8 & Endolysossome & Viruses & RNA & RIG-1, MDA5, NALP3 \\
\hline \multirow[t]{4}{*}{ TLR9 } & Endolysossome & Viruses & CpG-DNA & AlM2, DAl, IFl16 \\
\hline & & Bacteria & DNA & AlM2 \\
\hline & & Fungi & DNA & \\
\hline & & Parasites & Hemozoin (Plasmodium) & NALP3 \\
\hline TLR10 & Endolysossome & Unknown & Unknown & \\
\hline TLR11 & Plasma membrane & Protozoa & Profilin-like molecule & Unknown \\
\hline
\end{tabular}

After ligand binding, TLR signaling proceeds via two pathways: the myeloid differentiation factor 88 (MyD88)-mediated pathway, and the TIR-domaincontaining adaptor inducing an IFN- $\beta$ (TRIF)-mediated pathway. The former pathway causes the activation of the transcription factor NF- $\kappa \mathrm{B}$ and various mitogen-activated protein kinases (MAPK), which will activate several genes contributing to inflammatory reactions. The latter pathway causes induction of IFNs, whose stimulation leads cells to enter an antiviral state. TLR3 only activates the TRIF-mediated pathway, leading to the activation of the interferon regulatory factor (IRF)-3, an important transcription factor which induces the expression of type I IFNs and the indirect upregulation of IFN-dependent genes such as IFN-inducible protein 10 (IP-10) and inducible nitric oxide (iNOS). Moreover, TLR3 also activates NF- $\mathrm{NB}$, which upregulates the production of pro- inflammatory cytokines such as IL-1 $\beta$, IL-6 and TNF. TLR4 activates both pathways, activating NF- $\mathrm{BB}$ and inducing type I IFN production. All other TLRs activate exclusively the MyD88-dependent pathway $(13,43,44)$.

To sum up, the main functions of TLRs are to induce an inflammatory response and to establish adaptive immunity. TLRs activate tissue-resident macrophages to produce pro-inflammatory cytokines, including TNF, IL-1 $\beta$ and IL-6, which coordinate local and systemic inflammatory responses. These cytokines then activate surrounding cells to produce chemokines or adhesion molecules, thereby recruiting several inflammatory cells to the inflammation site. Recruited macrophages or neutrophils are activated and ingest invading pathogens through internalizing PRRs. Afterward, those cells kill the pathogens by producing nitric oxide, reactive oxygen species or defensins $(36,45)$. 
Several studies performed on animal models of TIDM have implicated TLR pathways in mechanisms linked with both diabetes induction and prevention. In both the NOD mouse and diabetes-prone BB rat, TLR upregulation can suppress diabetes. In the BioBreeding Diabetes Resistant rat; however, diabetes induced by virus infection involves the upregulation of TLR9 pathways, and generic TLR upregulation synergizes with virus infection on diabetes induction. Studies performed in mouse models of TIDM with spontaneous or induced diabetes implicate TLR1, TLR2, TLR3, and TLR7 in disease mechanisms [reviewed in (19)]. However, only a few studies analyzed the associations between polymorphisms in the TLR genes and susceptibility for TIDM. For example, Bjørnvold and cols. (46) reported that both TIDM and allergic asthma were significantly associated with the rs3804100 T allele in the TLR2 gene, and further associated with the haplotype including this polymorphism, possibly representing a susceptibility locus common for the two diseases. Park and cols. (47) reported that the $\mathrm{C} / \mathrm{C}$ genotype of the 1350 polymorphism in the TLR2 gene was associated with protection for TIDM. Moreover, the homozygous haplotype constituted by the T allele of the 597 polymorphism and the $\mathrm{C}$ allele of the 1350 polymorphism was associated with strong protection for TIDM. Cooper and cols. (48) analyzed a total of 1715 SNPs from the Wellcome Trust Case Control Consortium GWAS study of TIDM, and found an association between TIDM and a SNP in chromosome Xp22 (rs5979785), located 30kb centromeric from the TLR 7 and TLR 8 genes. The authors suggested that it is possible that this SNP, or variants in linkage disequilibrium with it, could alter TLR7 and/or TLR 8 expression and, therefore, modify the risk for TIDM.

\section{Toll-like receptor 3 (TLR3)}

Viral dsRNAs are potent inducers of type I IFN (IFN- $\alpha$ and IFN- $\beta$ ) antiviral responses and were reported to initiate their signaling via TLR3 (19). In addition to TLR3, dsRNA is recognized by the RLHs, RIG-I and melanoma differentiation-associated gene 5 (MDA5 or IFIHI). TLR3 and RIG-I/MDA5 differ in their cellular location and ligand specificities, and induce antiviral responses via different signaling pathways (34).

TLR3 is a type I transmembrane receptor with a molecular mass of $125 \mathrm{kDA}$ that has several distinct structural domains, as already mentioned (49). Dimerization of TLR3 is required for ligand binding, and dsRNA molecules must be at least 40 to $50 \mathrm{bp}$ in length in order to induce TLR3 signaling (49). TLR3 was originally identified to recognize a synthetic ana$\log$ of dsRNA, the polyinosinic:polyribocytidylic acid [poly(I:C)], which was found to be the most potent IFN inducer (34). TLR3 also recognizes genomic RNA from dsRNA viruses (including reoviruses) and dsRNA produced during replication of single-stranded RNA viruses (such as influenza A virus, encephalomyocarditis virus, and West Nile virus) and double-stranded DNA viruses (herpes simplex virus and murine cytomegalovirus) $(40,50)$. TLR3 can sense viral dsRNA in both the extracellular compartment and in endosomes. However, spatial localization seems to be important for recognition of viral dsRNA by TLR3, since cell-associated dsRNA has been found to be a more potent activator of TLR3 than soluble dsRNA (49).

The TLR3 gene is located on chromosome $4 \mathrm{q} 35$ (Figure 1), and comprises five exons with a $15,942 \mathrm{bp}$ transcript encoding a 3057bp mRNA and a 904 residue protein (5l). TLR $3 \mathrm{mRNA}$ has been detected in a number of human tissues including placenta, pancreas, lung, liver, heart, lymph nodes, and brain. Furthermore, TLR3 transcripts have been found in a variety of human and mouse immune cells, including $\mathrm{T}$ lymphocytes, natural killer cells, macrophages, mast cells and $\gamma \delta \mathrm{T}$ cells. $\mathrm{Hu}-$ man fibroblasts and epithelial cells express TLR3 both intracellularly and on the cell surface while monocytederived immature dendritic cells and myeloid dendritic cells only express TLR3 intracellularly, which demonstrates that cells utilize different strategies to sense viral invasion and initiate antiviral responses $(34,49)$.

In contrast to other TLR ligands, dsRNA signaling occurs via MyD88-independent pathways. For example, dsRNA binds TLR3 with the resultant recruitment of the adaptor molecule TRIF via a TIR-TIR domain interaction (Figure 2). Following the activation of endosomal TLR3 by dsRNA, TRIF co-localizes with TLR3 and dimerizes through its TIR domain and Cterminal region. This induces a conformational change that allows downstream signaling molecules to access their binding sites $(49,52)$. Then, TRIF recruits TNF receptor-associated factor 6 (TRAF6), forming a complex containing TGF- $\beta$-activated kinase-1 (TAKl), TAK-1 binding protein 2 (TAB2), and protein kinase $\mathrm{R}$, which mediates downstream NF- $\kappa \mathrm{B}$ activation. Protein kinase $\mathrm{R}$ also contributes to the dsRNA-induced activation of the p38 MAPK pathway by interaction with MAP kinase kinase 6 (MKK6). TRIF also recruits TRAF3, TRAF-family-member-associated NF- $\kappa$ B acti- 
vator binding kinase $\mathrm{l}(\mathrm{TBKl})$ and inducible IкB kinase (IKKi), which will phosphorilate IRF-3 and IRF-7 factors. After phosphorylation, activation and dimerization, IRF-3 and IRF-7 translocate into the nucleus and induce the expression of type I IFNs, which, after being secreted, will activate the expression of proinflammatory cytokines and chemokines by the signal transducers and activators of transcription 1 (STATl) pathway (41,53-55). This complex molecular response attracts immune cells, which will release more proinflammatory cytokines, such as IFN- $\gamma$, IL- $1 \beta$, and TNF. Local inflammation and activation of antiviral defenses seek to eradicate infection and trigger apoptosis of infected cells. However, in some genetically susceptible individuals, this defense system fails to work properly, instead inducing excessive, progressive inflammation and prolonged death of beta-cells, thus predisposing to the development of TIDM (2).

In this context, high levels of type I IFN are found in the pancreas of patients with TIDM (2), and IFN- $\alpha$ is known to contribute to the development of experimental viral-induced diabetes in mice $(56,57)$. However, recent data demonstrate that type I IFN production via TLR3 is critical to prevent diabetes caused by a virus with preferential tropism for pancreatic beta-cells in the NOD mouse, suggesting that viral infection and

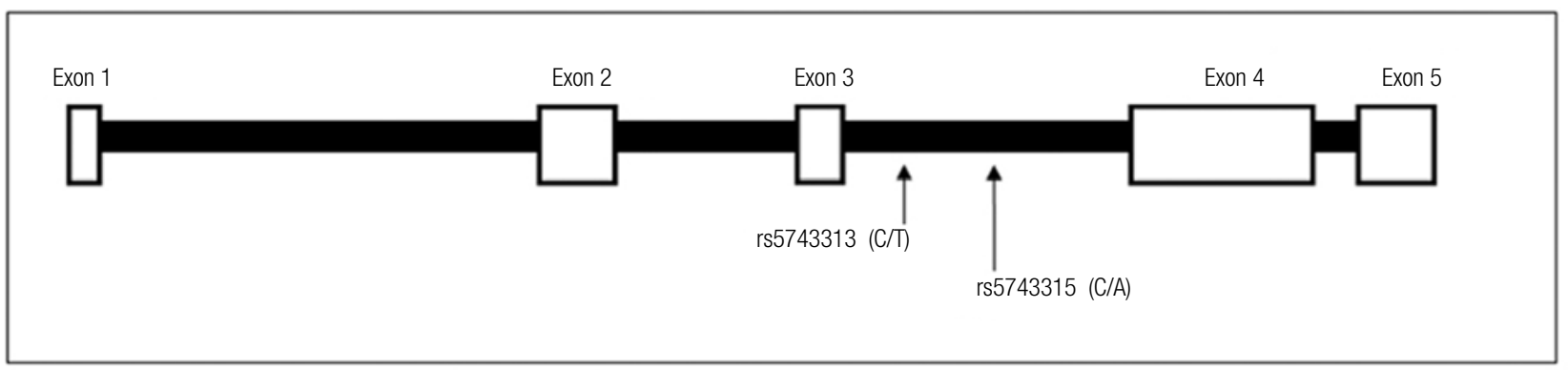

Figure 1. Map of Toll-like receptor (TLR3) locus on chromosome 4 (region 4q35). The five exons (boxes) are numbered from left to right according to the transcriptional region. The vertical arrows show the main common polymorphisms associated with type 1 diabetes mellitus.

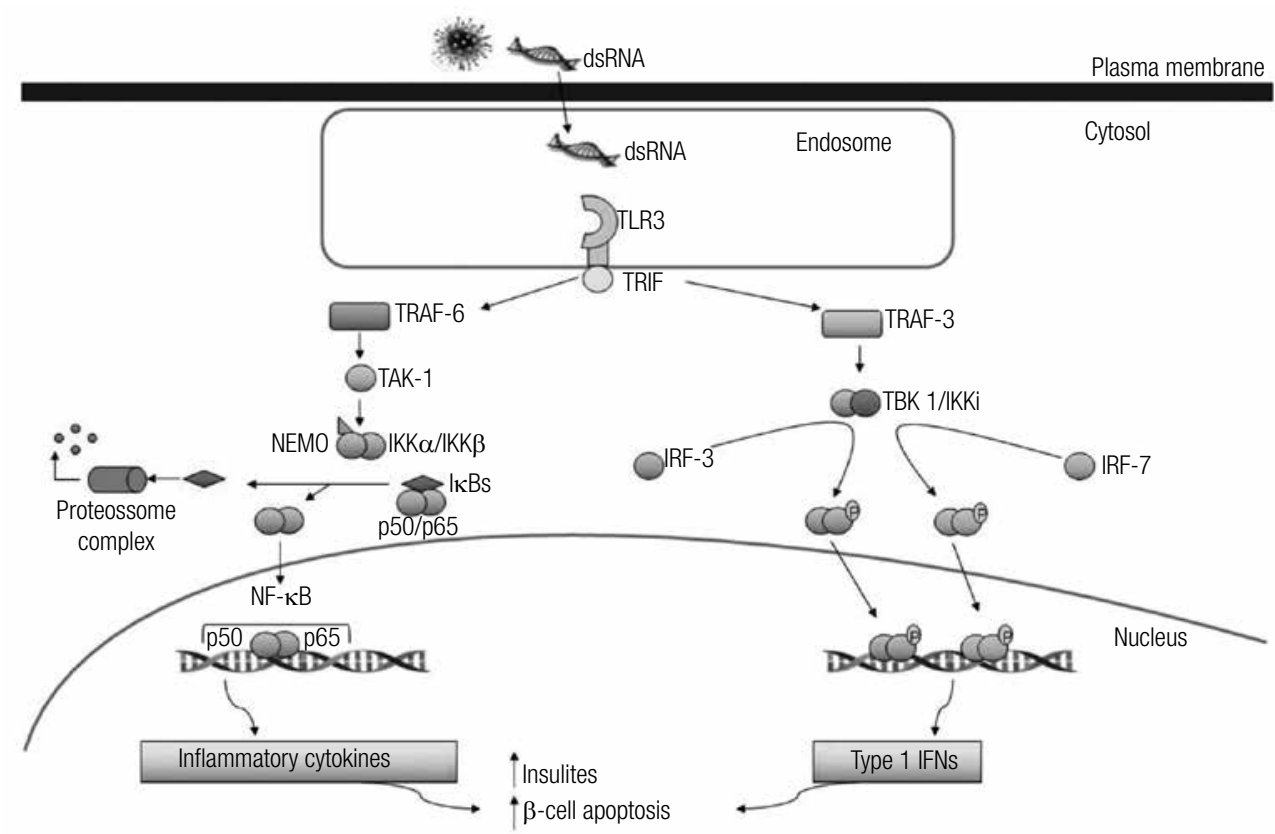

Figure 2. Toll-like receptor 3 (TLR3) signaling in pancreatic beta-cells. Viral double-stranded (dsRNA) binds TLR3 with the resultant recruitment of the adaptor molecule TRIF. Then, TRIF initiates two pathways via TRAF-6 and TRAF-3. TRAF- 6 activates TAK1. Activated TAK1 activates the IKK complex, which activates $\mathrm{NF}-\kappa \mathrm{B}$ subunits, which translocate to the nucleus. In the nucleus, NF- $\kappa \mathrm{B}$ triggers the transcription of several genes coding for proinflammatory cytokines and chemokines. TRAF3 activates TBK1/IKKi, which phosphorilate the transcription factors IRF-3 and IRF-7. After phosphorylation, activation and dimerization, IRF-3 and IRF-7, translocate to the nucleus and induce the expression of type 1 interferons, which, after being secreted, will also activate the expression of proinflammatory cytokines and chemokines. This complex molecular response attracts immune cells, which will release more proinflammatory cytokines, such as IFN- $\gamma, \mathrm{IL}-1 \beta$, and TNF, and might lead to the beta-cell apoptosis. Adapted from references Rasschaert and cols. (62) and Kumar and cols. (12). 
type I IFN responses may promote $\mathrm{T}$ cell tolerance by enhancing $\mathrm{T}$ regulatory cell function and up-regulation of inhibitory molecules and proinflammatory cytokines (50). It is known that the dsRNA-synthetic analog Poly(I:C) can accelerate diabetes in animal models, indicating that dsRNA sensors, such as TLR3, RIG-I and MDA5, can promote diabetes in an IFN-dependent manner $(58,59)$. Accordingly, human pancreatic islets infected with Coxsackie B5 virus or exposed to IFN- $\alpha$ or IFN- $\gamma+$ IL-1 $\beta$ exhibit increased expression of TLR3, RIG-I and MDA5 (60,61). Both intracellular and extracellular dsRNA may bind to TLR3 and trigger production of proinflammatory cytokines and chemokines, resulting in beta-cell apoptosis through the activation of NF-אB and IRF-3 (62-64).

Nevertheless, although TLR3 was the first dsRNA sensor identified as being able to activate NF- $\kappa B$ and IRF-3, its role as a primary antiviral receptor was recently called into question (65). In vivo antiviral responses against a wide range of viral pathogens were similar in wild-type and TLR 3 knockout mice (66). Indeed, more recent studies show that, whereas NF- $\mathrm{BB}$ and IRF-3 activation by extracellular dsRNA is TLR3-dependent, activation by intracellular dsRNA, a product of viral replication in the cytoplasm, also occurs through activation of RIG-I and IFIHI/MDA5 (63). These data indicate that a multiplicity of viral sensors seems essential for effective type I IFN response in beta-cells and other tissues. Distinct receptors differ in their specificity for viral products. Moreover, distinct viral receptors may differ in their tissue distribution or might be expressed at different time-points during infections. MDA5 is an IFN-induced protein, whereas TLR3 is constitutively expressed; therefore, TLR3-mediated antiviral responses might occur earlier than MDA5-mediated responses (50).

In brief, TLR3 is a plausible candidate for study in TIDM, because it reacts to viral products and is expressed in the pancreas and on antigen-presenting dendritic cells. Accordingly, Eleftherohorinou and cols. (67) developed a novel pathway-based method to assess the combined effect of more than 20,000 SNPs acting within 84 pathways associated with the innate and acquired immune responses to pathogens, and applied it to data from 14,000 subjects from United Kingdom with 7 common diseases, including TIDM. Variants responsible for the pathway association were identified and used to calculated predictive models for the diseases. These models were tested on an indepen- dent cohort from Northern Finland. They showed that multiple inflammatory pathways, containing 205 SNPs, were associated with TIDM. These SNPs, including SNPs in the pathway of TLR3, were found to be highly predictive of TIDM [91\% AUC (area under the receiver operating curve) in the UK sample and 79\% AUC in the Finnish cohort].

In addition, Pirie and cols. (51) reported that of nine TLR3 polymorphisms studied in a small sample of 153 subjects of Zulu descent from South Africa, a significant association with risk for TIDM was found for the major allele in the $2593 \mathrm{C} / \mathrm{T}$ (rs5743313) polymorphism and for the minor alleles in the $2642 \mathrm{C} / \mathrm{A}$ (rs5743315) and $2690 \mathrm{~A} / \mathrm{G}$ (novel) polymorphisms, which were found to be in complete linkage disequilibrium. However, correction of the P-values for the number of comparisons rendered the results no longer significant. Recently, a study from our group showed that the TLR 3 rs3775291 and rs13126816 polymorphisms seem to be associated with risk for TIDM, while the TLR3 rs5743313 and rs11721827 polymorphisms are associated with age at TIDM diagnosis and worst glycemic control in Brazilian subjects (68). Further studies are needed to investigate the association between TLR3 polymorphisms and TIDM in different populations.

\section{CONCLUSIONS}

TIDM is multifactorial disease associated with both genetic and environmental factors, and it is well known that viral infections play an important role in the development of TIDM in individuals with genetic predisposition. TLR3 recognizes viral replication-derived dsRNA, triggering the release of proinflammatory cytokines by immune system cells. This local inflammation and activation of antiviral defenses seeks to eradicate infection and trigger apoptosis of virus-infected cells. However, in some genetically susceptible individuals, this defense system fails to work properly, instead inducing excessive, progressive inflammation and prolonged death of beta-cells and thus predisposing to the development of TIDM. Hence, TLR3 is a good candidate gene for TIDM. However, although several studies have contributed to understanding the mechanisms underlying viral infection and TLR3 activity in TIDM pathogenesis, there are only a few studies reporting an association between TIDM and TLR3 gene polymorphisms. Thus, robust genetic studies are necessary in order to allow a better understanding of the role of the TLR3 gene in the susceptibility for TIDM. 
Knowledge of the factors associated with TIDM development will enable a keener understanding of its pathogenesis and may provide more effective approaches for the treatment and prevention of this disease.

Acknowledgements: this study was supported by grants from the Conselho Nacional de Desenvolvimento Científico e Tecnológico $(\mathrm{CNPq})$, Fundação de Amparo à Pesquisa do Estado do Rio Grande do Sul (FAPERGS), Coordenação de Aperfeiçoamento de Pessoal de Nível Superior (Capes) and Fundo de Incentivo à Pesquisa e Eventos (FIPE) at Hospital de Clínicas de Porto Alegre. D.C. and L.H.C. were recipients of scholarships from CNPq.

Disclosure: no potential conflict of interest relevant to this article was reported.

\section{REFERENCES}

1. American Diabetes Association. Diagnosis and classification of diabetes mellitus. Diabetes Care. 2013;36 Suppl 1:S67-74.

2. Eizirik DL, Colli ML, Ortis $\mathrm{F}$. The role of inflammation in insulitis and beta-cell loss in type 1 diabetes. Nat Rev Endocrinol. 2009;5(4):219-26.

3. Jahromi MM, Eisenbarth GS. Cellular and molecular pathogenesis of type 1A diabetes. Cell Mol Life Sci. 2007;64(7-8):865-72.

4. Kim MS, Polychronakos C. Immunogenetics of type 1 diabetes. Horm Res. 2005;64(4):180-8.

5. Steck AK, Rewers MJ. Genetics of type 1 diabetes. Clin Chem. 2011;57(2):176-85.

6. Todd JA, Walker NM, Cooper JD, Smyth DJ, Downes K, Plagnol $V$, et al. Robust associations of four new chromosome regions from genome-wide analyses of type 1 diabetes. Nat Genet. 2007;39(7):857-64

7. Maahs DM, West NA, Lawrence JM, Mayer-Davis EJ. Epidemiology of type 1 diabetes. Endocrinol Metab Clin North Am. 2010;39(3):481-97.

8. Coppieters KT, BoettlerT, von Herrath M. Virus infections in type 1 diabetes. Cold Spring Harb Perspect Med. 2012;2(1):a007682.

9. Knip M, Veijola R, Virtanen SM, Hyöty H, Vaarala O, Akerblom HK. Environmental triggers and determinants of type 1 diabetes. Diabetes. 2005;54 Suppl 2:S125-36.

10. Takeda K, Akira S. Toll-like receptors in innate immunity. Int Immunol. 2005;17(1):1-14.

11. Meylan E, Tschopp J, Karin M. Intracellular pattern recognition receptors in the host response. Nature. 2006;442(7098):39-44.

12. Kumar $\mathrm{H}$, Kawai T, Akira S. Pathogen recognition in the innate immune response. Biochem J. 2009;420(1):1-16.

13. Takeuchi $O$, Akira $S$. Pattern recognition receptors and inflammation. Cell. 2010;140(6):805-20.

14. Booth J, Wilson H, Jimbo S, Mutwiri G. Modulation of B cell responses by Toll-like receptors. Cell Tissue Res. 2011;343(1):131-40.

15. Arancibia SA, Beltran CJ, Aguirre IM, Silva P, Peralta AL, Malinarich $F$, et al. Toll-like receptors are key participants in innate immune responses. Biol Res. 2007;40(2):97-112.

16. Zarember KA, Godowski PJ.Tissue expression of human Toll-like receptors and differential regulation of Toll-like receptor mRNAs in leukocytes in response to microbes, their products, and cytokines. J Immunol. 2002;168(2):554-61.

17. Alexopoulou L, Holt AC, Medzhitov R, Flavell RA. Recognition of double-stranded RNA and activation of NF-kappaB by Toll-like receptor 3. Nature. 2001;413(6857):732-8.
18. Zipris D. Epidemiology of type 1 diabetes and what animal models teach us about the role of viruses in disease mechanisms. Clin Immunol. 2009;131(1):11-23.

19. Lien E, Zipris D. The role of Toll-like receptor pathways in the mechanism of type 1 diabetes. Curr Mol Med. 2009;9(1):52-68.

20. Filippi $\mathrm{CM}$, von Herrath MG. Viral trigger for type 1 diabetes: pros and cons. Diabetes. 2008;57(11):2863-71.

21. Akerblom HK, Vaarala O, Hyoty H, Ilonen J, Knip M. Environmental factors in the etiology of type 1 diabetes. Am J Med Genet. 2002;115(1):18-29.

22. Hyoty $H$, Hiltunen M, Knip M, Laakkonen M, Vahasalo P, Karjalainen J, et al. A prospective study of the role of coxsackie B and other enterovirus infections in the pathogenesis of IDDM. Childhood Diabetes in Finland (DiMe) Study Group. Diabetes. 1995;44(6):652-7.

23. Oikarinen $M$, Tauriainen $S$, Honkanen $T$, Oikarinen $S$, Vuori $K$, Kaukinen K, et al. Detection of enteroviruses in the intestine of type 1 diabetic patients. Clin Exp Immunol. 2008;151(1):71-5.

24. Elshebani A, Olsson A, Westman J, Tuvemo T, Korsgren O, Frisk G. Effects on isolated human pancreatic islet cells after infection with strains of enterovirus isolated at clinical presentation of type 1 diabetes. Virus Res. 2007;124(1-2):193-203.

25. Yeung WC, Rawlinson WD, Craig ME. Enterovirus infection and type 1 diabetes mellitus: systematic review and meta-analysis of observational molecular studies. BMJ. 2011;342:d35.

26. Jaidane $H$, Hober D. Role of coxsackievirus $B 4$ in the pathogenesis of type 1 diabetes. Diabetes Metab. 2008;34(6 Pt 1):537-48.

27. Tauriainen S, Oikarinen S, Oikarinen $\mathrm{M}, \mathrm{Hyoty} \mathrm{H}$. Enteroviruses in the pathogenesis of type 1 diabetes. Semin Immunopathol. 33(1):45-55.

28. Yoon JW, Austin M, Onodera T, Notkins AL. Isolation of a virus from the pancreas of a child with diabetic ketoacidosis. N Engl J Med. 1979;300(21):1173-9.

29. Dotta F, Censini S, van Halteren AG, Marselli L, Masini M, Dionisi $\mathrm{S}$, et al. Coxsackie B4 virus infection of beta cells and natural killer cell insulitis in recent-onset type 1 diabetic patients. Proc Natl Acad Sci U S A. 2007;104(12):5115-20.

30. Bach JF, Chatenoud L. The hygiene hypothesis: an explanation for the increased frequency of insulin-dependent diabetes. Cold Spring Harb Perspect Med. 2012;2(2):a007799.

31. Serreze DV, Ottendorfer EW, Ellis TM, Gauntt CJ, Atkinson MA. Acceleration of type 1 diabetes by a coxsackievirus infection requires a preexisting critical mass of autoreactive T-cells in pancreatic islets. Diabetes. 2000;49(5):708-11.

32. Tracy S, Drescher KM. Coxsackievirus infections and NOD mice: relevant models of protection from, and induction of, type 1 diabetes. Ann NY Acad Sci. 2007;1103:143-51.

33. Kariko K, Ni H, Capodici J, Lamphier M, Weissman D. mRNA is an endogenous ligand for Toll-like receptor 3. J Biol Chem. 2004;279(13):12542-50.

34. Amarante MK, Watanabe MA. Toll-like receptor 3: involvement with exogenous and endogenous RNA. Int Rev Immunol. 2010;29(6):557-73.

35. Chang JH, McCluskey PJ, Wakefield D. Toll-like receptors in ocular immunity and the immunopathogenesis of inflammatory eye disease. Br J Ophthalmol. 2006;90(1):103-8.

36. Kaisho T, Akira S. Toll-like receptor function and signaling. J Allergy Clin Immunol. 2006;117(5):979-87; quiz 88.

37. Kawai T, Akira S. Toll-like receptor and RIG-I-like receptor signaling. Ann NY Acad Sci. 2008;1143:1-20.

38. Moresco EM, LaVine D, Beutler B. Toll-like receptors. Curr Biol. 2011;21(13):R488-93.

39. Ishii KJ, Koyama S, Nakagawa A, Coban C, Akira S. Host innate immune receptors and beyond: making sense of microbial infections. Cell Host Microbe. 2008;3(6):352-63.

40. Kawai T, Akira S. Toll-like receptors and their crosstalk with other innate receptors in infection and immunity. Immunity. $2011 ; 34(5): 637-50$. 
41. Khoo JJ, Forster S, Mansell A. Toll-like receptors as interferonregulated genes and their role in disease. $\mathrm{J}$ Interferon Cytokine Res. 2011;31(1):13-25.

42. Akira S, Uematsu S, Takeuchi O. Pathogen recognition and innate immunity. Cell. 2006;124(4):783-801.

43. Yokota S, OkabayashiT, Fujii N.The battle between virus and host: modulation of Toll-like receptor signaling pathways by virus infection. Mediators Inflamm. 2010;2010:184328.

44. KawaiT, Akira S. TLR signaling. Cell Death Differ. 2006;13(5):816-25.

45. Medzhitov R. Recognition of microorganisms and activation of the immune response. Nature. 2007;449(7164):819-26.

46. Bjørnvold M, Munthe-Kaas MC, EgelandT, Joner G, Dahl-Jørgensen $\mathrm{K}, \mathrm{Njølstad} \mathrm{PR}$, et al. ATLR2 polymorphism is associated with type 1 diabetes and allergic asthma. Genes Immun. 2009;10(2):181-7.

47. Park Y, Park S, Yoo E, Kim D, Shin H. Association of the polymorphism for Toll-like receptor 2 with type 1 diabetes susceptibility. Ann NY Acad Sci. 2004;1037:170-4.

48. Cooper JD, Walker NM, Smyth DJ, Downes K, Healy BC, Todd JA. Follow-up of 1715 SNPs from the Wellcome Trust Case Control Consortium genome-wide association study in type I diabetes families. Genes Immun. 2009;10 Suppl 1:S85-94.

49. Yu M, Levine SJ. Toll-like receptor, RIG-I-like receptors and the NLRP3 inflammasome: key modulators of innate immune responses to double-stranded RNA viruses. Cytokine Growth Factor Rev. 2011;22(2):63-72.

50. Swiecki M, McCartney SA, Wang Y, Colonna M. TLR7/9 versus TLR3/MDA5 signaling during virus infections and diabetes. J Leukoc Biol. 2011;90(4):691-701.

51. Pirie FJ, Pegoraro R, Motala AA, Rauff S, Rom L, GovenderT, et al. Toll-like receptor 3 gene polymorphisms in South African Blacks with type 1 diabetes. Tissue Antigens. 2005;66(2):125-30.

52. Oshiumi H, Matsumoto M, Funami K, AkazawaT, SeyaT.TICAM-1, an adaptor molecule that participates in Toll-like receptor 3-mediated interferon-beta induction. Nat Immunol. 2003;4(2):161-7.

53. Yamamoto M, Sato S, Mori K, Hoshino K, Takeuchi O, Takeda K, et al. Cutting edge: a novel Toll/IL-1 receptor domain-containing adapter that preferentially activates the IFN-beta promoter in the Toll-like receptor signaling. J Immunol. 2002;169(12):6668-72.

54. Sato S, Sugiyama M, Yamamoto M, Watanabe Y, Kawai T, Takeda $\mathrm{K}$, et al. Toll/IL-1 receptor domain-containing adaptor inducing IFN-beta (TRIF) associates with TNF receptor-associated factor 6 and TANK-binding kinase 1, and activates two distinct transcription factors, NF-kappa B and IFN-regulatory factor-3, in the Tolllike receptor signaling. J Immunol. 2003;171(8):4304-10.

55. Taniguchi T, Takaoka A. The interferon-alpha/beta system in antiviral responses: a multimodal machinery of gene regulation by the IRF family of transcription factors. Curr Opin Immunol. 2002;14(1):111-6.
56. Stewart TA, Hultgren B, Huang X, Pitts-Meek S, Hully J, MacLachIan NJ. Induction of type I diabetes by interferon-alpha in transgenic mice. Science. 1993;260(5116):1942-6.

57. Alba A, Puertas MC, Carrillo J, Planas R, Ampudia R, Pastor X, et al. IFN beta accelerates autoimmune type 1 diabetes in nonobese diabetic mice and breaks the tolerance to beta cells in nondiabetes-prone mice. J Immunol. 2004;173(11):6667-75.

58. Moriyama H, Wen L, Abiru N, Liu E, Yu L, Miao D, et al. Induction and acceleration of insulitis/diabetes in mice with a viral mimic (polyinosinic-polycytidylic acid) and an insulin self-peptide. Proc Natl Acad Sci U S A. 2002;99(8):5539-44.

59. Devendra D, Jasinski J, Melanitou E, Nakayama M, Li M, Hensley B, et al. Interferon-alpha as a mediator of polyinosinic:polycytidylic acid-induced type 1 diabetes. Diabetes. 2005;54(9):2549-56.

60. Hultcrantz M, Huhn MH, Wolf M, Olsson A, Jacobson S, Williams $\mathrm{BR}$, et al. Interferons induce an antiviral state in human pancreatic islet cells. Virology. 2007;367(1):92-101.

61. Ylipaasto P, Kutlu B, Rasilainen S, Rasschaert J, Salmela K, Teerijoki $\mathrm{H}$, et al. Global profiling of coxsackievirus- and cytokine-induced gene expression in human pancreatic islets. Diabetologia. 2005;48(8):1510-22.

62. Rasschaert J, Ladrière L, Urbain M, Dogusan Z, Katabua B, Sato S, et al. Toll-like receptor 3 and STAT-1 contribute to double-stranded RNA+ interferon-gamma-induced apoptosis in primary pancreatic beta-cells. J Biol Chem. 2005;280(40):33984-91.

63. Dogusan Z, Garcia M, Flamez D, Alexopoulou L, Goldman M, Gysemans $C$, et al. Double-stranded RNA induces pancreatic betacell apoptosis by activation of the toll-like receptor 3 and interferon regulatory factor 3 pathways. Diabetes. 2008;57(5):1236-45.

64. Liu D, Cardozo AK, Darville MI, Eizirik DL. Double-stranded RNA cooperates with interferon-gamma and IL-1 beta to induce both chemokine expression and nuclear factor-kappa B-dependent apoptosis in pancreatic beta-cells: potential mechanisms for viral-induced insulitis and beta-cell death in type 1 diabetes mellitus. Endocrinology. 2002;143(4):1225-34.

65. Meylan E,Tschopp J.Toll-like receptors and RNA helicases: two parallel ways to trigger antiviral responses. Mol Cell. 2006;22(5):561-9.

66. Edelmann KH, Richardson-Burns S, Alexopoulou L, Tyler KL, Flavell RA, Oldstone MB. Does Toll-like receptor 3 play a biological role in virus infections? Virology. 2004;322(2):231-8.

67. Eleftherohorinou $H$, Wright $V$, Hoggart $C$, Hartikainen $A L$, Jarvelin MR, Balding D, et al. Pathway analysis of GWAS provides new insights into genetic susceptibility to 3 inflammatory diseases. PLoS ONE. 2009;4(11):e8068.

68. Assmann TS, Brondani Lde A, Bauer AC, Canani LH, Crispim D. Polymorphisms in theTLR3 gene are associated with risk for type 1 diabetes mellitus. Eur J Endocrinol. 2014;170(4):519-27. 